\title{
The distribution of blood and gas within the lungs measured by scanning after administration of ${ }^{133} \mathrm{Xe}$
}

\author{
C. T. DOLLERY AND P. M. S. GILLAM \\ From the Department of Medicine, Postgraduate Medical School, Hammersmith Hospital, London
}

Many diseases of the lung or the pulmonary circulation cause regional differences in function within the lungs that may be of both diagnostic and therapeutic importance. The use of the radioactive gases ${ }^{15} \mathrm{O}$ and ${ }^{133} \mathrm{Xe}$ has enabled detailed studies of regional lung function to be made that were not possible with earlier techniques (Dyson, Hugh-Jones, Newbery, Sinclair and West, 1960 ; Ball, Stewart, Newsham, and Bates, 1962). These gases are inhaled or injected into the lungs and their distribution is detected by counters arranged outside the chest. The precision of the information gained depends upon the accuracy and uniformity of the counting fields and the number of counters used. Up to the present, counters have been used either as pairs arranged in front and behind the chest or singly in a number of different positions. Single counters require less apparatus to survey a number of different areas, but pairs wired in parallel are preferable because they give a more uniform counting field stretching through the lung. The sensitivity of a single counter falls off rapidly with distance. As it is often desirable to study the function of several areas of lung on the same breath, a formidable array of electronic equipment may be required.

The problem is essentially how to display the distribution of radioactivity within the lung, which is a large three-dimensional object. Threedimensional display is not practical at present, but either a scintillation camera or a scanning method could be used to give a two-dimensional picture. The scintillation camera (Anger and Rosenthal, 1959) consists of a large crystal or crystals which act in the same manner as the film in a camera with a pinhole collimator or similar simple focusing device. The image on the crystal is detected by an array of photomultiplier tubes and displayed on a cathode ray tube. It has not yet proved possible to apply this method to the lung but in the future it may have promise. At present it is more practical to scan the source of radioactivity by moving the counters over it (Brownell,
1959). The shape of the lung, which is very much longer in the vertical plane than in any other, means that a single vertical scan is able to detect most abnormalities of functional importance. In this paper we describe the development and use of a profile scanning method which gives more information about the topographical distribution of lung function than can be obtained with quite complex arrays of stationary counters.

\section{APPARATUS AND METHODS}

The methods used for handling and dispensing radioactive xenon have been published (Dollery, HughJones, and Matthews, 1962). Pairs of sodium iodide crystals $1 \frac{1}{2}$ in. long $\times 1$ in. thick were used with lead collimators 6 in. long with an aperture of $1 \frac{1}{2}$ in. at the crystal tapering to $\frac{1}{2}$ in. at the external orifice. Using these collimators with a lung model uniformly filled with ${ }^{133} \mathrm{Xe}, 87 \%$ of counts are contributed from a cylinder $9 \mathrm{~cm}$. in diameter.

Each pair of counters was mounted on a rigid metal frame to ensure accurate alignment. The frame slid vertically on two metal pillars, and movement was controlled by a hydraulic jack mounted between the pillars. The hydraulic ram, which was reversible, was driven by an electric pump, and the flow of fluid was regulated by a valve that allowed movement up or down under power, and a neutral position which recirculated the fluid to the pump (Fig. 1). Each pair of counters could be moved independently but, for scanning, they were always used coupled together so that all four moved as a single unit.

Scans were usually made with the counters moving vertically and the patient sitting upright in a chair. However, a special couch could be used for scans while the patient lay flat and the counters moved horizontally. The motors could move the counters at any speed up to $3.8 \mathrm{~cm}$./ second.

The signals from the counters were amplified and fed to ratemeters and displayed on a twin pen recorder with a central marker pen which showed a pulse every half-inch the counters moved. ${ }^{13 s} \mathrm{Xe}$ emits $80 \mathrm{KeV}$ gamma rays and $30 \mathrm{KeV} X$ rays, and the bias of the amplifiers was adjusted to filter out the $30 \mathrm{KeV}$ peak and with it some of the scattered radiation. 


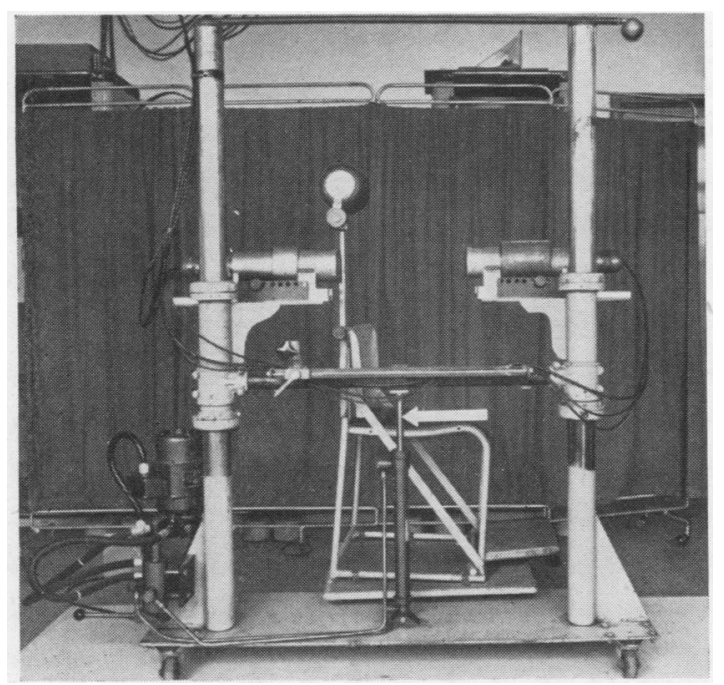

FIG. 1. The apparatus used for scanning. The chair and one pair of counters are shown. The hydraulic ram (arrowed) moves the pair of counters over the chest from base to apex. The electric pump and control valve are at the bottom left.

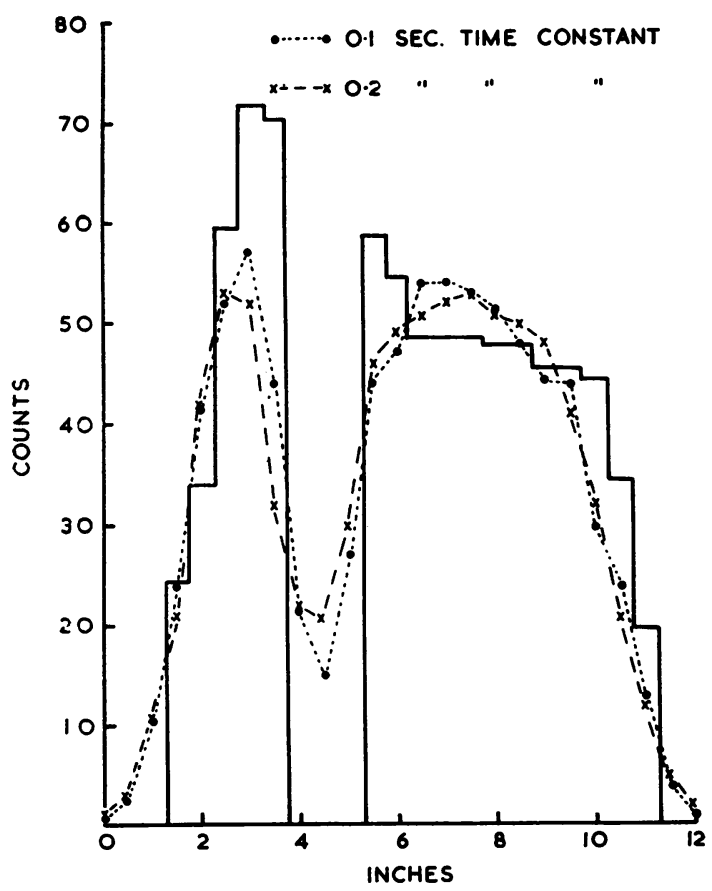

FIG. 2. Scans made at a speed of $3.75 \mathrm{~cm} . / \mathrm{sec}$. over a stack of sources (solid line) with a dip to zero in its lower part. There is little difference in the shape of the scan obtained with recording apparatus with an overall time constant of $0.1 \mathrm{sec}$. and alternative apparatus with a time constant of $0.2 \mathrm{sec}$.
RESOLUTION The resolution of the counters was tested using two different shaped sources filled with ${ }^{133} \mathrm{Xe}$. One consisted of flat boxes $20 \times 20 \times 1.4 \mathrm{~cm}$. and the other, glass test tubes $15 \times 1.25 \mathrm{~cm}$. closed with a rubber bung. Each type was scanned at low speed (less than $0.5 \mathrm{~cm}$./ sec.) first as a single source and then as a pair containing approximately equal amounts of radioactivity. The pairs of sources were gradually separated and the scans repeated. From these data the resolution can be calculated in two ways ; first, the width of the scan of a single source at half the peak height, and, second, the distance separating two sources when it first becomes possible to distinguish two distinct peaks. Both methods and both types of source gave a resolution of $3.6 \mathrm{~cm}$. Further studies were made with the tubular type of source which was easier to make in large numbers.

The pen recorder and ratemeters used previously for radioactive gas work had an overall time constant of 0.2 second. Modifications were made to a second pair of ratemeters and a special pen recorder was fitted to decrease the time constant to 0.1 second. The two recording systems were compared by scanning a known distribution of 20 tubes containing ${ }^{133} \mathrm{Xe}$ stacked one above the other. When the stacks were scanned at gradually increasing speeds up to the maximum of $3.75 \mathrm{~cm}$. $/ \mathrm{sec}$. there was little difference between the recording systems (Fig. 2).

A COMPARISON WITH STATIONARY COUNTERS The sensitivity of the scanning system was compared with stationary counters by constructing a stack of sources with a sharp dip to zero within it. Three stationary counter positions were used spaced out over the model, and the information obtained was compared with the known distribution of the counts and with the scan (Fig. 3). When one of the stationary counter pairs was directly over the gap it was clearly detected, but when the gap was midway between two pairs they failed to show it clearly. By contrast the scan, which covered the whole of the lung, always revealed the defect.

VALIDITY OF BLOOD FLOW, VENTILATION, AND VOLUME RATIOS DERIVED FROM SCANS Radioactive xenon is used in lung function studies to compare ventilation and blood flow in different regions. The counting rate in each area after a single tidal breath or an intravenous injection of dissolved xenon gives a measure of the amount entering that region in gas or blood, but to make comparisons it is helpful to know the lung volume in each counting field. The lung volume calibration is made using the counting rate in each area after rebreathing a ${ }^{133} \mathrm{Xe}$ mixture with air from a closed-circuit spirometer until it has equilibrated.

If the lung is scanned after an intravenous injection of dissolved ${ }^{133} \mathrm{Xe}$ and again after rebreathing, the two curves represent the distribution of blood flow and lung volume at each level within the limits of resolution of the system. If the ratios of the heights of the two curves are taken, say every $\frac{1}{2}$ in., a plot can be 

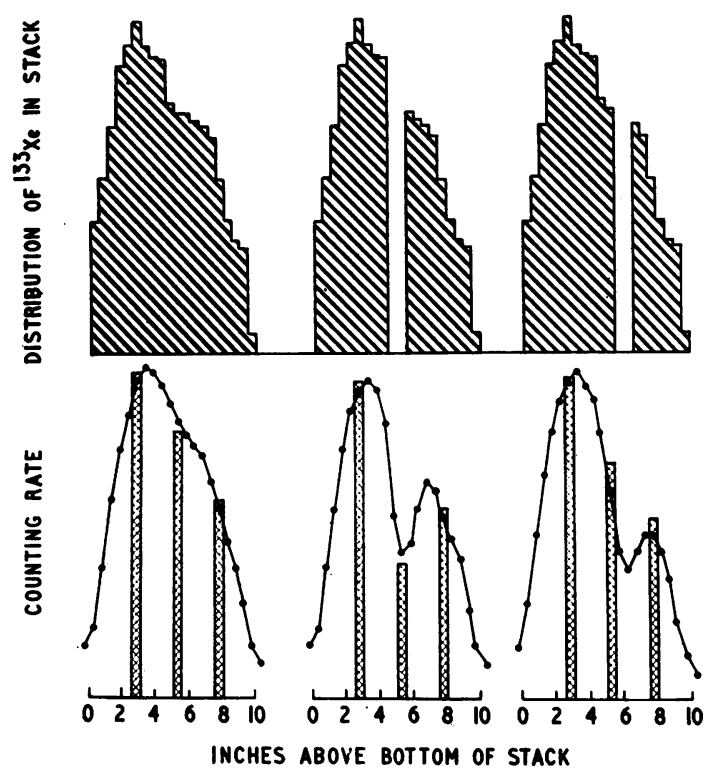

A. $\quad$ B. .

FIG. 3. (Above) The histogram indicates the distribution of radioactivity in the lung models; (below) the continuous -lines show the curves obtained by scanning and the hatched columns readings obtained with stationary counters. When one stationary cou tter is over the gap (B) it gives a clear indication of it but when the gap is between two counters $(C)$ they fail to detect it. In both instances the scan gives a satisfactory indication of the position of the gap.

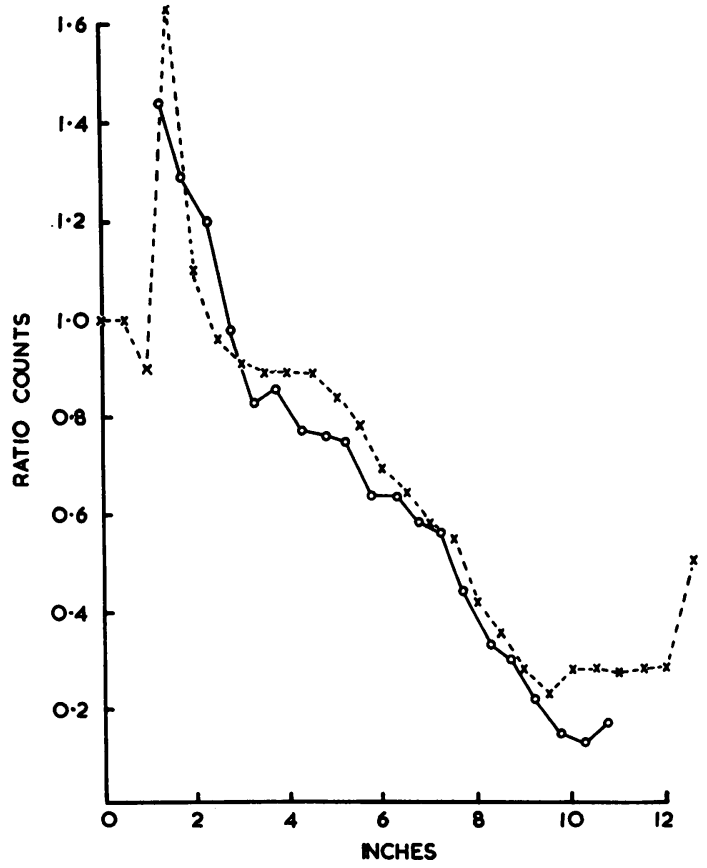

FIG. 5. The ratio of the known distribution of the sources. in Fig. 4 is shown by the continuous line $(\mathrm{O}-\mathrm{O})$ and the ratio calculated from the scans by the dotted line $(\mathrm{x}----\mathrm{x})$. Over most of the range the agreement is close.

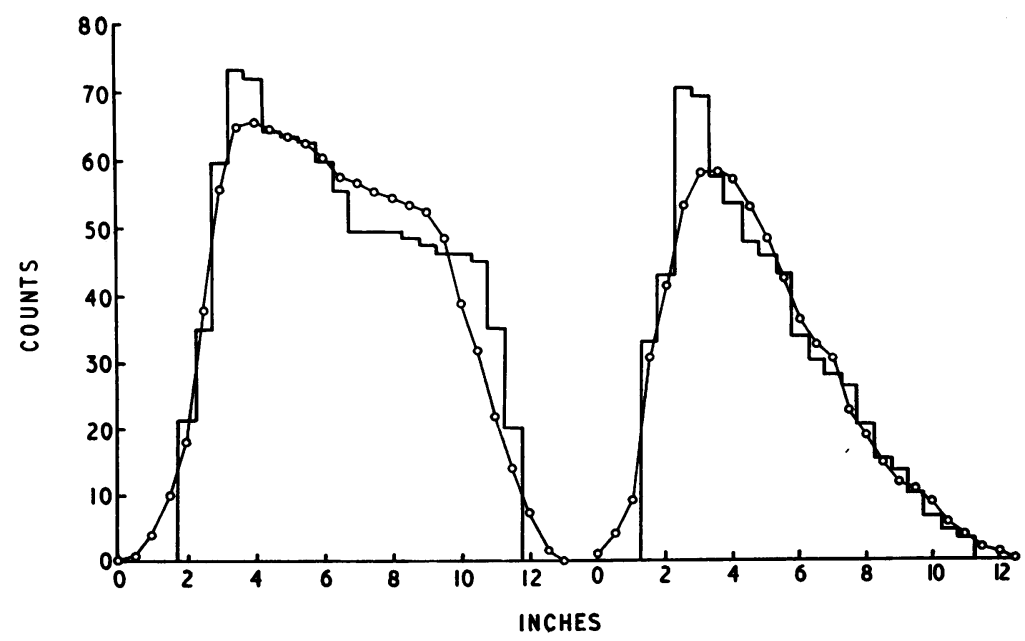

FIG. 4. Scans of two stacks of sources chosen to represent the lung after an injection of ${ }^{138} X e$ dissolved in saline and after equilibration on a closed circuit. The histogram shows the distribution of the sources and the continuous line the scan from the counters. For comparison each pair of histograms and scans have been scaled to an equal area. 
made of the flow per unit lung volume up the lung. A similar method is used with the scans after a tidal breath to make ventilation/volume and ventilation/ blood flow comparisons. To test the validity of using the scans in this way two stacks of sources were made, one to simulate blood flow and the other volume distribution in the upright normal lung (Fig. 4). The ratio of 'flow/volume' was derived from the two scans and also from the known ratios of the distribution of radioactivity in the two stacks of sources (Fig. 5). The two agree closely except at the extremes where the counters were at the limits of the stacks of sources.

\section{CLINICAL APPLICATIONS}

Clinical lung scans are made with the patients sitting upright in a chair and with the counters at rest over the lowermost part of the chest. The blood flow measurement is made after injection of about 1 millicurie of ${ }^{133} \mathrm{Xe}$ dissolved in saline through a fine (PE 60) polythene catheter passed from a needle in an arm vein into the superior vena cava. Five seconds after injection the patient is asked to start breathing slowly in and then to hold his breath. The scan is made 10 seconds after the injection is complete and it takes about six seconds. A further scan is made at 30 seconds after the end of the breath-holding period to determine the rapidity of wash-out of the injected ${ }^{133} \mathrm{Xe}$ from the lungs. The ventilation measurement is made during breath holding after a single tidal breath in of ${ }^{133} \mathrm{Xe}$ in a concentration of 1 to 2 millicuries per litre from a spirometer. A scan is made at 30 seconds after the end of breath holding to measure the wash-out.

Rebreathing studies are made for two purposes; to calibrate the scans for the lung volume in the field at each level and to examine the wash-in and wash-out of poorly ventilated areas. The patient breathes ${ }^{133} \mathrm{Xe}$ at a concentration of 1 to 2 millicuries per litre from a closed-circuit spirometer, and scans are made during breathing at 30 seconds after starting and then at every minute after starting. If ventilation is normal the lungs and spirometer reach equilibrium after about one and a half minutes but if there are poorly ventilated areas, as in bullous emphysema, it may be impossible to reach equilibrium in a few minutes. In a patient with poorly ventilated areas rebreathing is continued for four to six minutes and at the end a scan is made with the patient breath holding in inspiration. Further scans are made during normal air breathing, at minute intervals, as the ${ }^{133} \mathrm{Xe}$ is washed out of the lungs. At the end of the procedure the counters are set opposite the second rib and a note is made of the height above the bottom of the scan. This enables functional abnormalities to be related to structural changes shown on the chest radiograph.

With the amounts of radioactivity used in these studies and the counting apparatus described, the counting rate is about 300 counts per second after the injection and the tidal breath and 1,000 c.p.s. after equilibration. The radiation dose does not exceed 300 millirads to the lungs in a patient with poor ventilation in some areas, and if ventilation is normal the dose is slightly less (Cyclotron Unit Technical Memo No. 84).

The scan curves can be analysed in several ways. The simplest method is to take the ratios of deflection at standard points on each scan to map out the distribution of perfusion/volume, ventilation/volume or ventilation/perfusion. By assuming that the whole of both lungs are scanned the total area under each scan can be taken as $100 \%$ of perfusion, ventilation, and volume respectively, and a plot can be made in terms of the percentage of the total of each appearing in a given amount of the lung. The wash-in and wash-out scans can be analysed either by taking the deflections at different times to draw a curve of the change in activity with time at different positions or, more simply, to trace and superimpose curves to gain an approximate idea of the ventilation characteristics of different areas.

Some examples of the applications of the method and the different methods of analysis follow.

DISTRIBUTION OF FUNCTION IN THE NORMAL LUNG

The scans in Fig. 6 were made from a 27-year-old doctor who had been sitting at rest in a chair for 10 minutes. The striking feature is that the peak of the scan following injection of ${ }^{133} \mathrm{Xe}$ ('perfusion') is much lower down the chest than either the one taken after a tidal breath ('ventilation') or after equilibration on a closed circuit ('volume'). The ratios of perfusion/volume, ventilation/volume, and ventilation/perfusion are shown in Figure 7. To facilitate comparison each scan has been scaled to the same area so that in each case the ratio for the whole lung is unity. The blood flow per unit of lung volume is five times higher at the base of the lung than at the apex but the ventilation is much more evenly distributed. In consequence the ventilation/ perfusion ratio is nearly five times higher at the apex than at the base. The distribution of function is similar to that found by West and Dollery (1960) using $\mathrm{C}^{15} \mathrm{O}_{2}$. The scan allows a topographical 


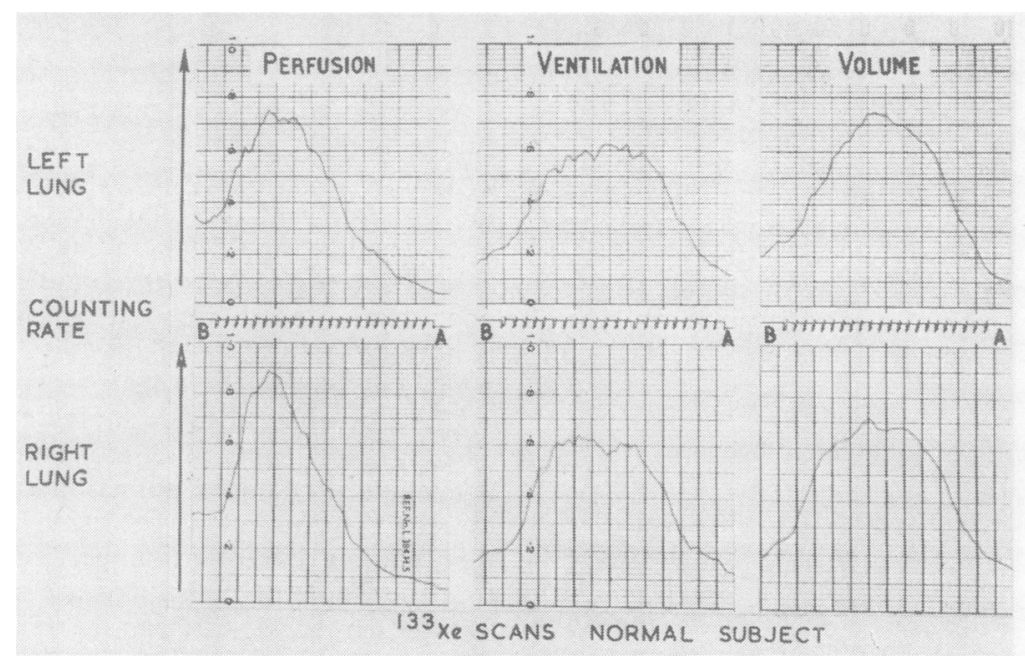

FIG. 6. Scans in a normal subject after a ${ }^{133} X e$ injection (perfusion), a tidal breath of ${ }^{133} \mathrm{Xe}$ (ventilation), and after rebreathing the isotope on a closed circuit until equilibrated (volume). The centre marker indicates displacement every half inch from base $(B)$ to apex $(A)$. Note the peak is much lower down the chest after the injection than after either the tidal breath or equilibration.
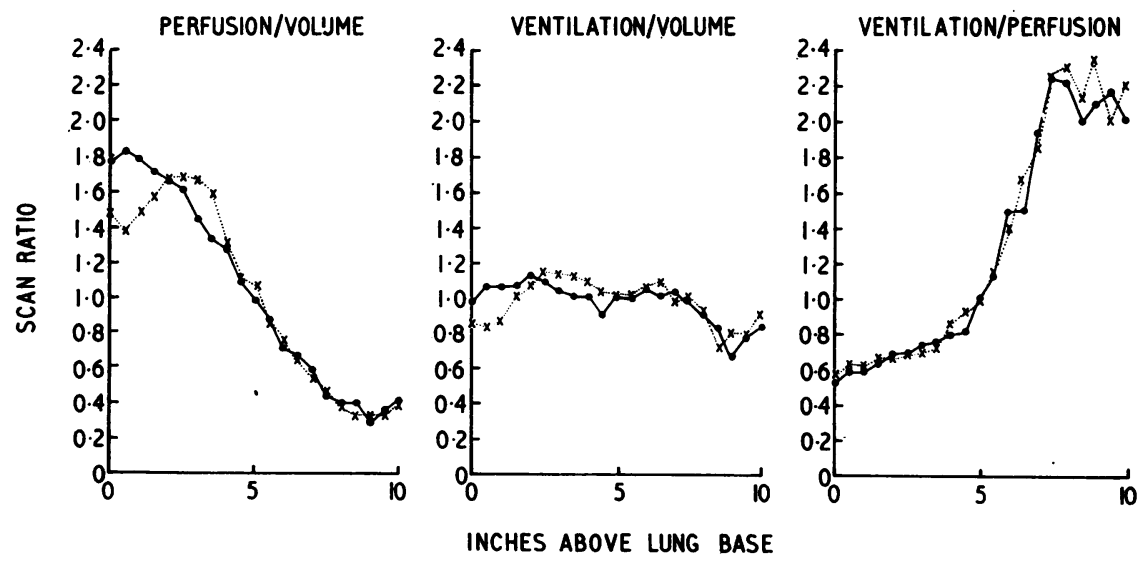

FIG. 7. Distribution of perfusion/volume, ventilation/volume, and ventilation/perfusion calculated from the scans of a normal subject in Fig. 6. The perfusion per unit volume is five to six times higher at the base than at the apex. The ventilation per unit volume is only slightly higher at the base than at the apex; in consequence the ventilation/perfusion ratio is four to five times higher at the apex than at the base (-— left lung; $\mathrm{x}-\mathrm{x}$ right lung). 
FIG. 8. Chest radiograph of a 58-year-old man with breathlessness on slight exertion. The diaphragms are low but there is no regional abnormality in the lung fields.
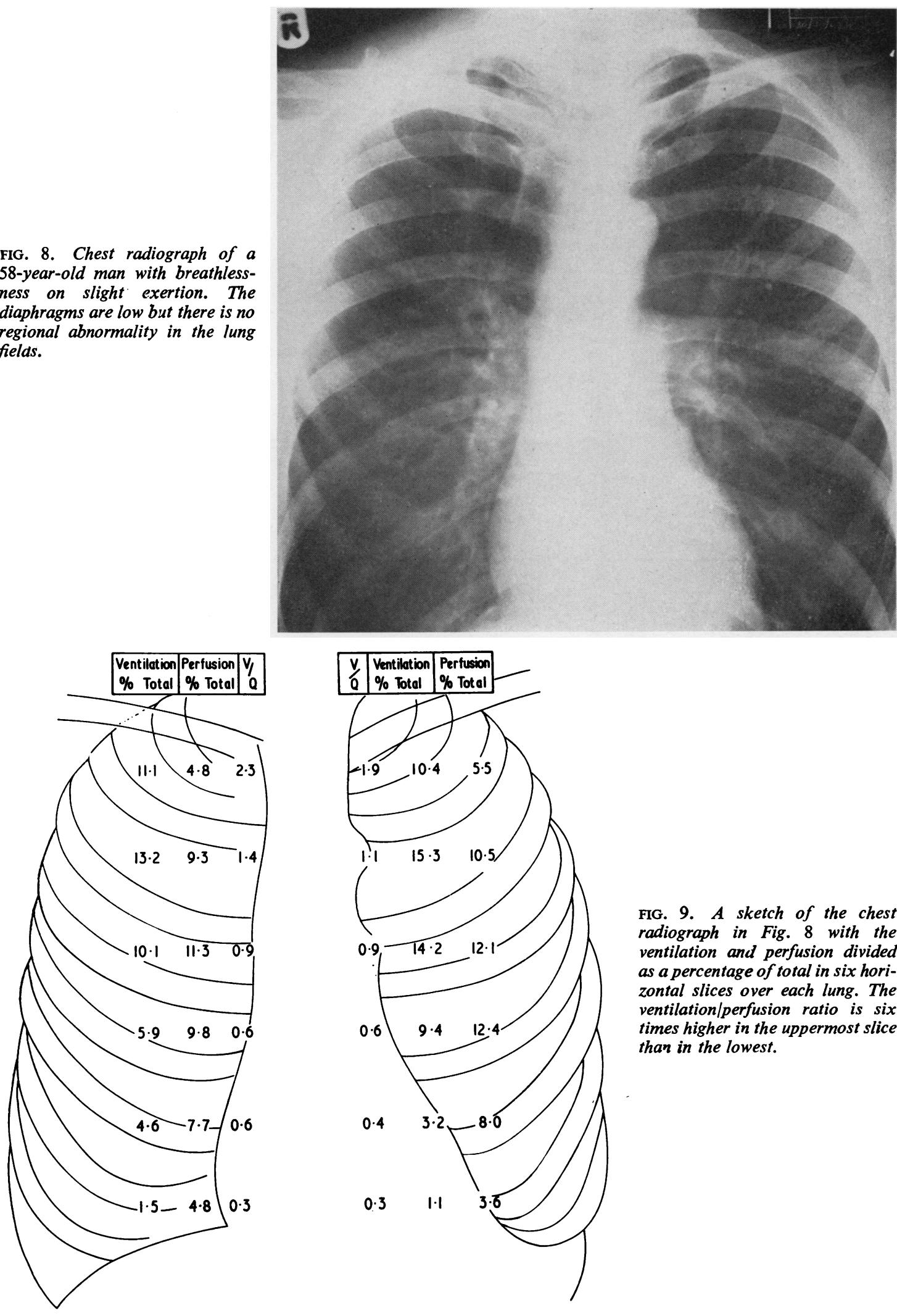

FIG. 9. A sketch of the chest radiograph in Fig. 8 with the ventilation and perfusion divided as a percentage of total in six horizontal slices over each lung. The ventilation/perfusion ratio is six times higher in the uppermost slice than in the lowest. 
FIG. 10. Scans of ventilation (o-0) and perfusion $(\mathrm{x}--\mathrm{x})$ redrawn to equal areas in the patient whose radiograph is Fig. 8. The perfusion is evenly distributed but the ventilation is preponderantly distributed to the upper zones. The ventilation/perfusion ratio (-—) is 10 times higher in the upper part of the left lung than in the lower.
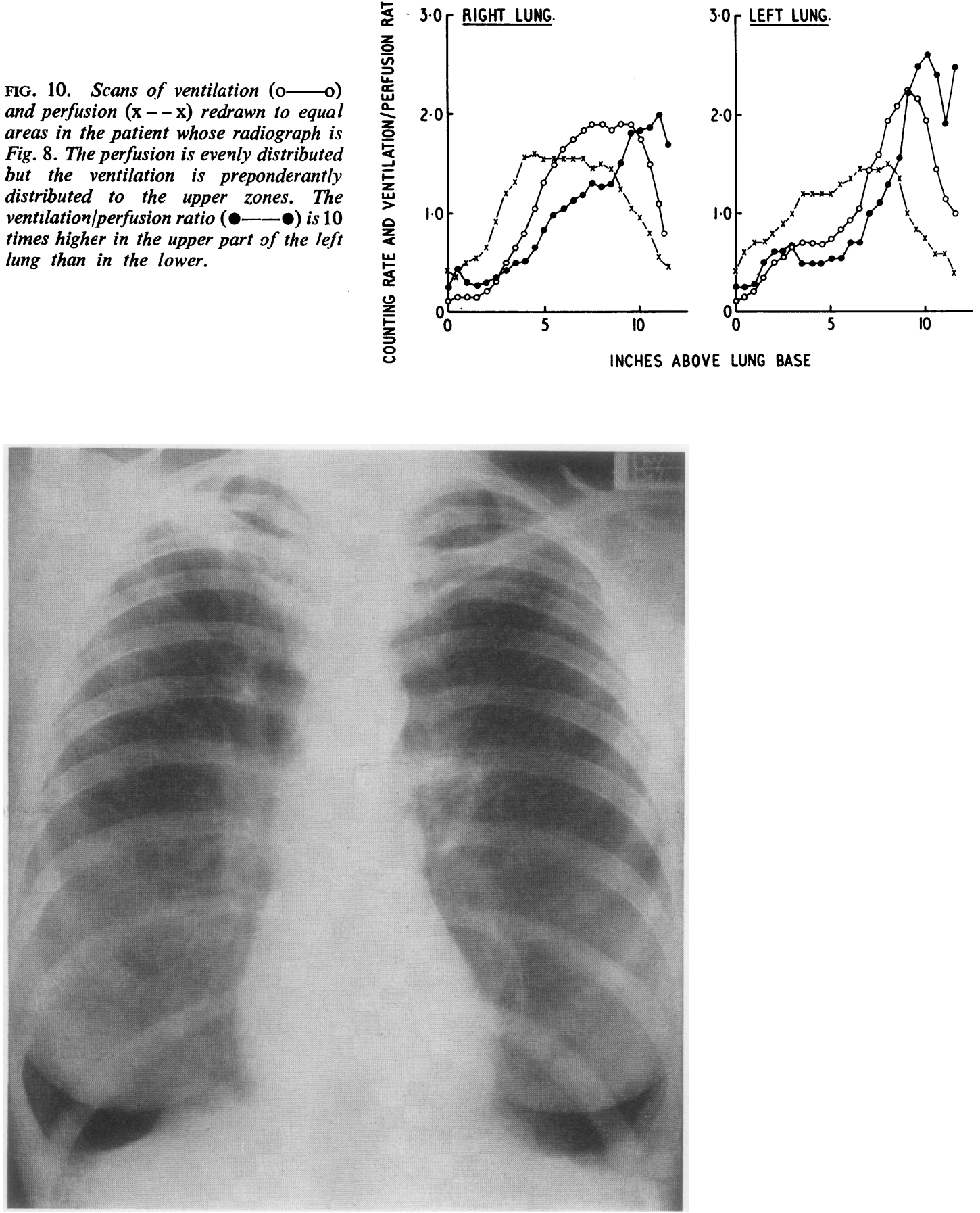

FIG. 11. Chest radiograph of a woman of 55 showing bilateral basal bullae, larger on the left than the right. 


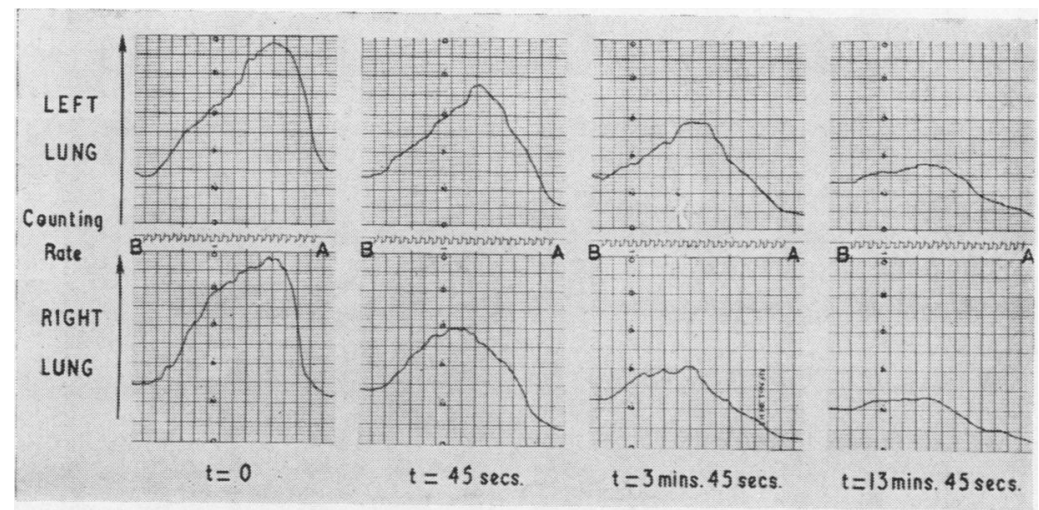

FIG. 12. Scans made sequentially during air breathing after six minutes rebreathing ${ }^{133} \mathrm{Xe}$ by the patient whose radiograph is Fig. 11. More radioactivity had entered the apical regions $(A)$ than the base $(B)$ at the end of the period of rebreathing, labelled $t=0$. During air breathing the wash-out was much faster at the apex so that after $13 \frac{3}{4}$ minutes more was left at the base despite its initially low activity.
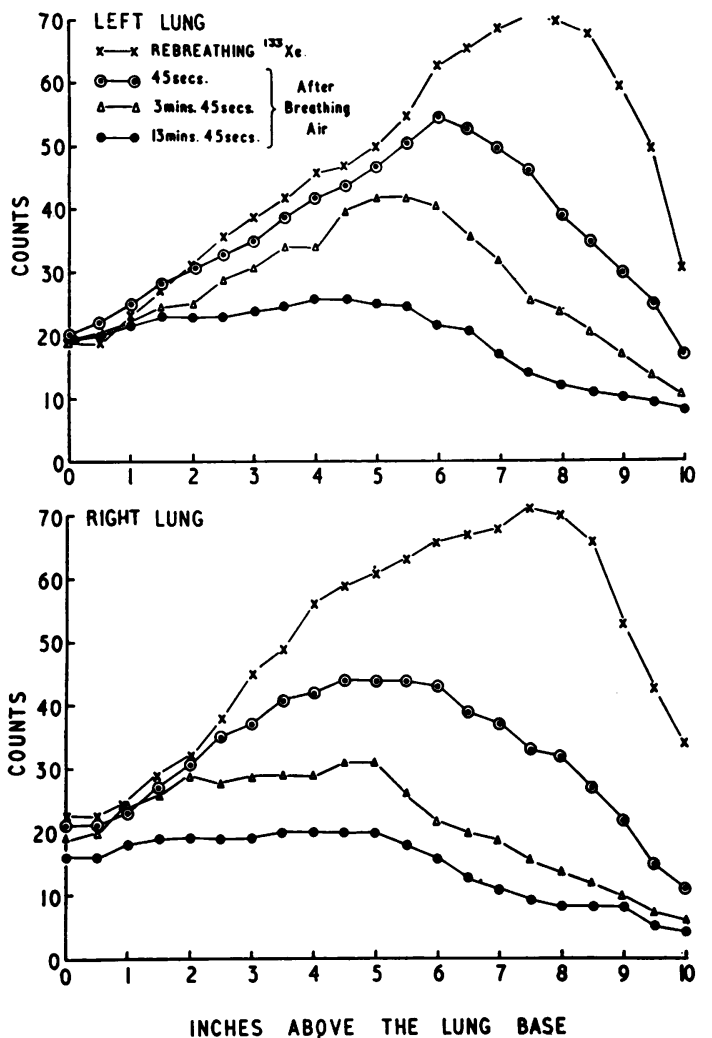

FIG. 13. The scans of Fig. 12 replotted on to a common scale. Where the scan lines are wide apart, as at the apex, the wash-out is faster whereas where they are crowded together the wash-out is very slow (after allowing for the difference in initial deflection). assessment of function in a single study whereas methods using stationary counters usually require pooling of several studies to produce a similar detailed profile of the distribution of blood flow and ventilation.

EMPHYSEMA AND BRONCHITIS The first patient, a man of 58, had a 15-year history of bronchitis and had become increasingly breathless over two years. He was able to walk only a few paces before he became dyspnoeic. His chest radiograph showed only minor bullous changes in the midzones with flat and low diaphragms. The scans revealed that $74 \%$ of the total ventilation was going to the upper half of the lungs which were receiving $53 \%$ of the blood flow. The lower half of the chest received only $27 \%$ of the ventilation but had $46 \%$ of the blood flow (Fig. 9). The ventilation and perfusion scans on which these results are based are shown drawn to equal areas in Figure 10. By displaying the chest radiograph and function divided up into 2-in. thick slices of lung on the same scale it is possible to appreciate the distribution of function in relation to anatomical features on the radiograph (Fig. 8).

Another patient, a woman aged 55 years, was disabled by breathlessness on slight exertion. Her radiograph showed bilateral bullae at the lung bases (Fig. 11). The ventilation and perfusion scans both showed little function in the bullous zone, and ventilation and perfusion were reasonably well balanced in contrast to the previous patient. During rebreathing, little ${ }^{133} \mathrm{Xe}$ entered the bullae and what did was very slowly removed during air breathing. By replotting the traces during wash- 
out on to a common graph, it is possible to build up a contour map of ventilatory function (Figs. 12 and 13). As some ${ }^{133} \mathrm{Xe}$ is taken up into the blood during breathing and is slowly returned to the lungs from the tissues, the wash-in and washout plots are not purely a measure of alveolar ventilation. However, for clinical purposes the differences between poorly ventilated and more normal areas are usually so great as to make this factor relatively unimportant.

MITRAL STENOSIS Changes in pulmonary vascular pressures cause alterations in the normal distribution of blood flow within the lungs, and one of the most remarkable changes that takes place is that of severe mitral stenosis. In this condition the blood flow is reduced so that in severe cases it may be much less than in the upper zones of the lungs. There is no corresponding change in ventilation (Dollery and West, 1960). The patient whose pulmonary angiogram is shown in Fig. 14 was a woman aged 56 years who had extremely severe mitral stenosis with a resting pulmonary artery pressure of $90 / 27 \mathrm{~mm}$. Hg. The scans for the

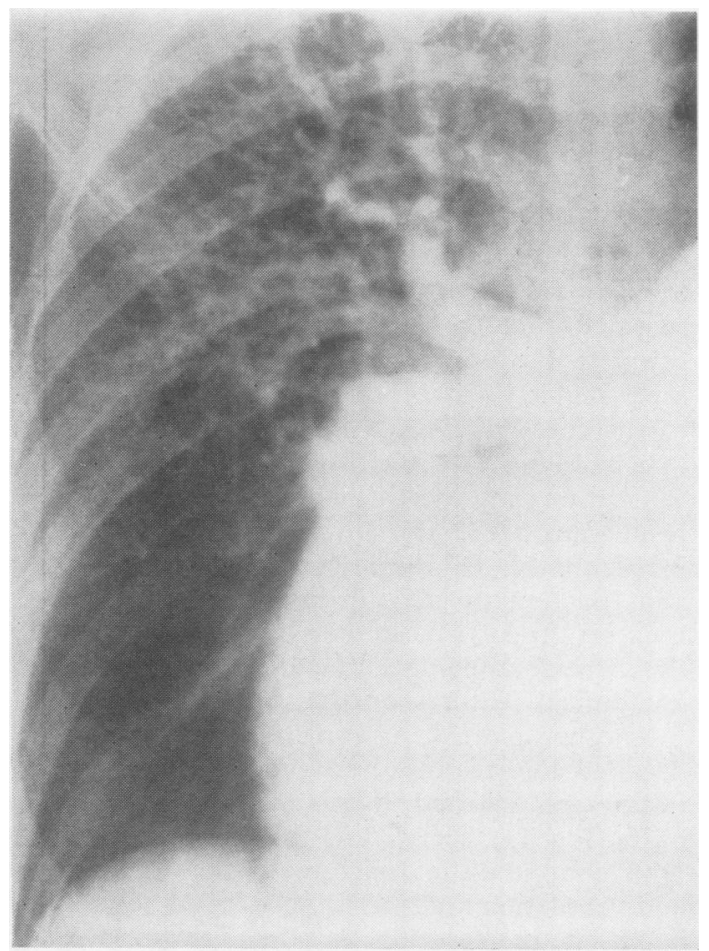

FIG. 14. Pulmonary angiogram of the right lung of $a$ patient with severe mitral stenosis. The blood vessels are attenuated at the lung base. right lung are shown alongside the arteriogram to show that the blood flow per unit volume was more than four times higher at the apex than at the base whereas ventilation was evenly distributed throughout (Fig. 15).
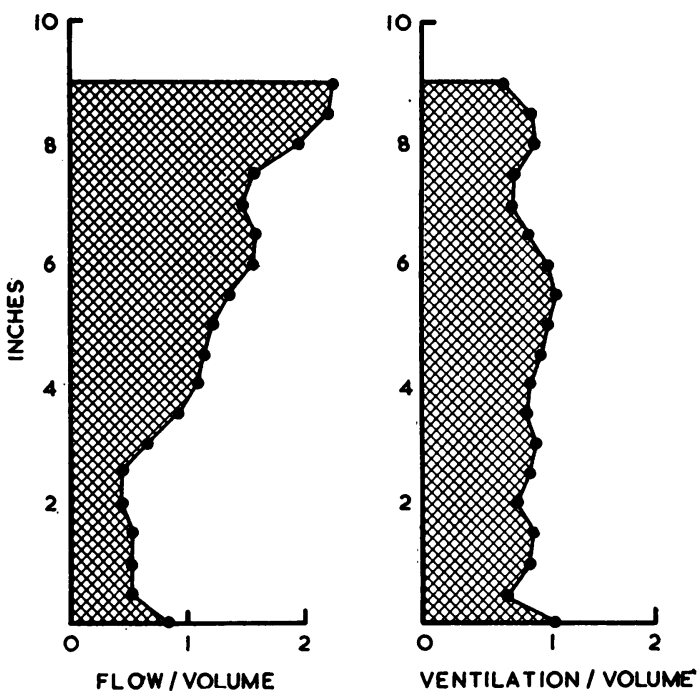

FIG. 15. The distribution of blood flow and ventilation per unit volume in the right lung of the patient with mitral stenosis whose pulmonary angiogram is Figure 14. The vertical scale shows the height above the lung base and the horizontal one is an arbitrary ratio scaled to unity for the whole lung. The blood flow per unit volume is four times higher in the upper part of the lung while ventilation is evenly distributed.

\section{DISCUSSION}

The objective of studies of regional lung function is to map out the distribution of ventilation and blood flow within the lungs so as to produce a functional equivalent of the chest radiograph. Radioactive gas studies have made a substantial contribution towards realizing this objective because of the ease with which they can be used and the small areas that can be studied.

The scanning method has some substantial advantages over the use of a complex array of stationary counters to examine many areas on a single breath or injection. First it gives a continuous picture of the distribution of function from top to bottom of the lung and thus reduces the possibility of missing a lesion because it did not fall within the field of a counter. Another important advantage is the economy of apparatus that it makes possible. Two pairs of scintillation counters with their associated amplifiers, rate- 
meters, and recorders supplemented by $£ 250$ of hydraulic equipment will do more than can be done with six or eight pairs of stationary counters which, with their ancillary equipment, would cost several thousand pounds more.

The scanning method is suitable for recording distribution following intravenous injection and a tidal breath and is suitable for wash-in and washout studies if the scans are repeated frequently.

There are several possible sources of error. As the ratios are taken from scans done at different times it is important to ensure, as far as possible, that the patient is in the same position and at the same lung volume for each of them. The chair used is designed to prevent changes in posture by use of a head and backrest but close attention to position is essential. The easiest way to reach the same lung volume in disabled patients is to ask them to breathe in as deeply as they can for each scan.

For the perfusion measurement it is important that the patient should not close the glottis and do Valsalva's manoeuvre while the ${ }^{133} \mathrm{Xe}$ is arriving in the lung. This difficulty is overcome by asking him to breathe in slowly, and once the radioactivity has been evolved into the alveolar gas it does not matter if the patient does strain against a closed glottis during breath holding.

In patients with bullous emphysema some areas of lung may be very poorly ventilated and equilibration only very slowly reached during rebreathing. Rebreathing cannot be continued for more than six minutes because of radiation dosage, and this may not be long enough to reach equilibrium. Under these circumstances the 'volume' scan will be too small, and ventilation and perfusion related to this volume will be overestimated. Results of ventilation and perfusion scans in such patients are best expressed as a percentage of the total for the whole lung going to particular areas. Volume figures derived from radiographs would be an alternative method if it were important to relate ventilation and perfusion to volume.

The scanning method could be used in any body position although our apparatus has only been used with the chest upright or lying flat. Most patients have to be studied with the chest upright because they are too breathless when lying down.

The method has proved useful in evaluating patients who have regional abnormalities of function, and it is a very easy test for the patient to perform. The applications are similar to those of the earlier ${ }^{133} \mathrm{Xe}$ procedure (Ball et al., 1962 ; Dollery et al., 1962) and include a wide variety of diseases of the lungs and the pulmonary circulation. As the scans give a more complete picture of function, judgments about the proportion of blood flow and ventilation entering different areas can be made with greater certainty.

\section{SUMMARY}

A method has been devised of using pairs of collimated crystal scintillation counters, driven by hydraulic rams, to scan the lungs after intravenous injection or inhalation of ${ }^{133} \mathrm{Xe}$.

Tests with a lung model show that the resolutio. of the counters for point sources is $3.6 \mathrm{~cm}$. and that when the counters are propelled at $3.8 \mathrm{~cm} . /$ sec. an overall time constant of the recording apparatus of $0.2 \mathrm{sec}$. is adequate.

The scan records the profile of distribution of radioactivity in the vertical plane, and tests with known sources show good agreement between the real and calculated distributions.

In clinical studies the scans can be used to map out the distribution of blood flow and ventilation and to record the rate of wash-in and wash-out during rebreathing.

The method gives more information with less apparatus than is required for a complex array of stationary counters.

We thank Mr. G. Forse for skilled technical assistance. We are grateful to Dr. P. Hugh-Jones for his support and to members of the staff of the M.R.C. Cyclotron Unit, particularly Mr. D. D. Vonberg and Dr. C. M. E. Matthews, for advice and facilities.

\section{REFERENCES}

Anger, H. O., and Rosenthal, D. J. (1959). In Medical Radioisotope Scanning, p. 59. International Atomic Energy Agency, Vienna. (Proceedings of a seminar organized by I.A.E.A. and W.H.O.) Ball, W. C., Stewart, P. B., Newsham, L. G. S., and Bates, D. V. (1962). Regional pulmonary function studied with xenon. J. clin. Invest, 41, 519.

Brownell, G. L. (1959). In Medical Radioisotope Scanning, p. 1. International Atomic Energy Agency, Vienna. (Proceedings of a seminar organized by I.A.E.A. and W.H.O.).

Dollery, C. T., Hugh-Jones, P., and Matthews, C. M. E. (1962). Use of radioactive xenon for studies of regional lung function. A comparison with oxygen-15. Brit. med. J., 2, 1006 . comparison with oxygen-15. Brit. med. J., 2, 1006. carbon monoxide and carbon dioxide in the lungs of patients with mitral stenosis. Circulat. Res., 8, 765.

Dyson, N. A., Hugh-Jones, P., Newbery, G. R., Sinclair, J. D., and West, J. B. (1960). Studies of regional lung function using radioactive oxygen. Brit. med. J., 1, 231.

West, J. B., and Dollery, C. T. (1960). Distribution of blood flow and ventilation-perfusion ratio in the lung, measured with radioactive $\mathrm{CO}_{2}$.J. appl. Physiol., 15, 405. 